\title{
A NOTE ABOUT WIENER-HOPF SETS
}

\author{
GÉRARD LETAC
}

Definition. A Wiener-Hopf set is a subset $S$ of the real line $R$ such that

(i) $S^{+}$and $S^{-}$are not empty,

(ii) $S^{+}+S^{-} \subset S$,

where $S^{+} \cup S^{0} \cup S^{-}$is the usual decomposition of $S$ into positive, negative and null parts.

Let us explain this definition: Considering a probability measure $\mu$ on $R$, the Wiener-Hopf decomposition of $\mu$ is given by:

$$
\epsilon_{0}-\mu=(1-\alpha)\left(\epsilon_{0}-\mu^{-}\right) *\left(\epsilon_{0}-\mu^{+}\right)
$$

where the star indicates convolution, $\epsilon_{0}$ is the unit mass at $0,0 \leqq \alpha \leqq 1$, and $\mu^{+}$and $\mu^{-}$are positive measures of mass not larger than one concentrated on $R^{+}=\{x: x>0\}$ and $R^{-}=\{x: x<0\}$. The measure $\mu^{-}$(resp. $\mu^{+}$) has a probabilistic interpretation as a distribution of the first visit to $R^{-}$(resp. $R^{+}$) of the random walk $S_{n}=X_{1}+\cdots+X_{n}$ where $X_{1}, \cdots, X_{n}$ are independent random variables with the same distribution $\mu$; the number $\alpha$ is the probability that the first visit to $R^{+} \cup\{0\}$ (or $R^{-} \cup\{0\}$ ) is to 0 . See Feller [1] for details.

(1) can be rewritten:

$$
\mu=\alpha \epsilon_{0}+(1-\alpha)\left(\mu^{+}+\mu^{-}-\mu^{+} * \mu^{-}\right)
$$

This formula gives us two examples of Wiener-Hopf sets.

Proposition 1. If $\mu\left(R^{+}\right)$and $\mu\left(R^{-}\right)$are positive the support of $\alpha \epsilon_{0}+\mu^{+}+\mu^{-}$is a Wiener-Hopf set.

Proof. Let us denote by $S(\nu)$ the support of any measure $\nu$, that is to say the smallest closed subset of $R$ carrying the whole mass of $\nu$. If $S^{+} \cup S^{0} \cup S^{-}=S=S\left(\alpha \epsilon_{0}+\mu^{+}+\mu^{-}\right)$is the decomposition on $R^{+}, 0$, $R^{-}$of $S$, we have $S\left(\mu^{+}\right) \supset S^{+}$and $S\left(\mu^{-}\right) \supset S^{-}$. $\mu$ positive implies, by (2), $S \supset S\left(\mu^{+} * \mu^{-}\right)$. But $S\left(\mu^{+} * \mu^{-}\right) \supset S\left(\mu^{+}\right)+S\left(\mu^{-}\right)$. Then $S^{+}+S^{-} \subset S$. Since by hypothesis $S^{+}$and $S^{-}$are not empty, $S$ is a Wiener-Hopf set.

Let us denote by $\nu_{a}$ the atomic part of any measure $\nu$.

Proposition 2. If $\mu_{a}\left(R^{+}\right)$and $\mu_{a}\left(R^{-}\right)$are positive the set of atoms of $\alpha \epsilon_{0}+\mu^{+}+\mu^{-}$is a Wiener-Hopf set.

Received by the editors September 18, 1968. 
PRoof. (2) implies $\mu_{a}=\alpha \epsilon_{0}+(1-\alpha)\left(\mu_{a}^{+}+\mu_{a}^{-}-\mu_{a}^{+} * \mu_{a}^{-}\right)$and the proof goes in a similar way to that in Proposition 1.

We are led to consider Wiener-Hopf sets from the following observed fact: Suppose $S(\mu)$ is a set of integers. Then $S\left(\alpha \epsilon_{0}+\mu^{+}+\mu^{-}\right)$ has no holes, that is to say, it is an interval (not necessarily bounded) of integers. A similar property holds when $S(\mu)$ is not concentrated on lattice points.

The aim of this note is to provide the proof of these results in the following theorem:

TheOREM. If $S$ is a Wiener-Hopf set, let $\rho=\inf \left\{x: x \in S^{+}\right\}$. Either $\rho>0$ and $S / \rho$ is an interval of integers, or $\rho=0$ and the closure $\bar{S}$ of $S$ is an interval of $R$.

Let us introduce two useful notations: $[x, y]_{z}$ is the interval of integers with end points $x$ and $y$. When $x<0$ and $y>0$, let $A(x, y)$ $=[x, y] \cap\{m x+n y: m, n$ nonnegative integers, $m+n>0\}$. We need now three lemmas:

LEMma 1. If $p$ and $q$ are positive integers such that $(p, q)=1$, then $A(-p, q)=[-p, q]_{z}$

Proof. Easy, using Bezout identity.

Lemma 2. If $x / y$ is irrational, $A(x, y)$ is dense in $[x, y]$.

Proof. Easy, using the fact that multiples of a positive irrational number, taken modulo 1 , are dense in $[0,1]$.

Lemma 3. $x \in S^{-}$and $y \in S^{+}$imply $A(x, y) \subset S$.

Proof. We use induction on $N=m+n$. Suppose that $m x+n y \in S$ for any $m$ and $n$ such that $x \leqq m x+n y \leqq y$ and $m+n<N$. This is true for $N=2$. If $x \leqq m x+n y \leqq y$ and $m+n=N \geqq 2$ then $m \geqq 1, n \geqq 1$, $(m-1) x+n y \geqq 0$ and $m x+(n-1) y \leqq 0$. Clearly, at least one of these two numbers (say, the first) is in $[x, y]$ and by the induction hypothesis, is in $S$. Hence either $(m-1) x+n y \in S^{0}$ and $m x+n y=x \in S$, or $(m-1) x+n y \in S^{+}$and $m x+n y \in S^{-}+S^{+} \subset S$.

Proof of the Theorem. Suppose $\rho>0$. We claim first $\rho \in S^{+}$. If not, there exists a strictly decreasing sequence $\left(y_{n}\right)$ in $S$ such that $y_{n} \underset{n \infty}{\longrightarrow} \rho$. Since $S^{-}$is not empty choose $x \in S^{-}$. If there existed $n$ such that $x / y_{n}$ is irrational, by Lemmas 2 and $3 \rho$ would be 0 . Hence $x / y_{n}$ is rational for any $n$, and there exist $\lambda_{n}>0$ and positive integers $p_{n}$ and $q_{n}$ such that $\left(p_{n}, q_{n}\right)=1$ and $x=-\lambda_{n} p_{n}$ and $y_{n}=\lambda_{n} q_{n}$. But the $x / y_{n}=p_{n} / q_{n}$ being distinct, the sequence $q_{n}$ is not bounded, and there exists a sequence $n_{k}$ of integers such that $q_{n_{k}} \underset{\mathbf{k} \infty}{\longrightarrow} \infty$ and $\lambda_{n_{k}} \rightarrow 0$. By 
Lemma 1, $A\left(x, y_{n_{k}}\right)=\lambda_{n_{k}}\left[-p_{n_{k}}, q_{n_{k}}\right]_{z}$ and, by Lemma 3, $\rho=0$. Hence $\rho>0$ implies $\rho \in S^{+}$.

Choose $x \in S^{-}$. We show that $x=-n \rho$ where

$$
n+1=\min \left\{k: x+k \rho \in S^{+}\right\} \text {. }
$$

By Lemma 3, $x+n \rho \in S^{-} \cup S^{0}$. If $x+n \rho \in S^{-}$, then $x+(n+1) \rho<\rho$, a contradiction. Hence $x+n \rho \in S^{0}$. Furthermore $0 \in S, A(x, \rho)$ $=\rho[-n, 1] z \subset S$ and $-\rho=\max \left\{x: x \in S^{-}\right\}$. Using the same proof one can show that every element of $S^{+}$is a multiple of $\rho$, and $S / \rho$ is an interval of $Z$.

Suppose $\rho=0$. Let $x \in S^{-}, \epsilon>0$ and $t$ be such that $x<t<0$. We claim that $(t-\epsilon, t) \cap S^{-}$is not empty. There exists a strictly decreasing sequence $\left(y_{n}\right)$ in $S^{+}$such that $y_{n} \underset{n \infty}{\longrightarrow} 0$. Define $k_{n}$, nonnegative integer, and $\epsilon_{n}$, by $t-x=k_{n} y_{n}+\epsilon_{n}$ with $0 \leqq \epsilon_{n}<y_{n}$. If $y_{n}$ is such that $y_{n}<\epsilon$ then $x+k_{n} y_{n} \in(t-\epsilon, t)$. But by Lemma $3, x+k_{n} y_{n} \in S^{-}$. Hence $S^{-}$is dense in $[x, 0]$. The proof for $S^{+}$goes in the same way and the closure of $S$ is an interval of $R$.

\section{BIBLIOGRAPHY}

1. W. K. Feller, An introduction to probability theory and applications, Vol. II, Wiley, New York, 1966.

University OF MONTREal 